\title{
De novo ring chromosome 3: a new case with a mild phenotype
}

\author{
M McKinley, A Colley, P Sinclair, D Donnai, T Andrews
}

\begin{abstract}
We report an 18 year old female with a de novo ring chromosome 3 found after investigation for short stature. Her karyotype was interpreted as $46, \mathrm{XX}$, $r(3)(p 26.2 q 29)$. Her phenotype is milder than previously reported cases and illustrates the mild end of the spectrum of the ring chromosome 3 phenotype.
\end{abstract}

There are only five published cases of ring chromosome 3 , but in the first two reports ${ }^{12}$ the breakpoints were undefined. In the other three reports ${ }^{3-5}$ the breakpoints on the short arm were more proximal than in this case, although the long arm breakpoints were identified as q29 in all cases. We compared reports of subjects with ring chromosome 3 and those with terminal deletions of chromosome $3 p$ and $3 q$ with this female, looking for a distinctive phenotype or evidence of the ring syndrome.

\section{Case report}

The female proband was the second child of healthy, non-consanguineous parents; the maternal age was 22 years and paternal age 24 years at delivery. The first child was a normal male and there was no relevant family history. After a normal pregnancy and delivery, birth weight was $2950 \mathrm{~g}$ at term and there were no neonatal problems. Her subsequent course, including developmental milestones, was normal apart from significant growth retardation. There was normal pubertal development and menarche was at the age of 11 years. She attended a normal school but received special help.

At the age of 18 years she was investigated for short stature. Her OFC was $48 \mathrm{~cm}$ (below the $3 \mathrm{rd}$ centile), height $145 \mathrm{~cm}(-3.5 \mathrm{SD}$ below mean) with normal

Department of Medical Genetics, St Mary's Hospital, Hathersage Road, Manchester M13 0JH.

M McKinley, A Colley, P Sinclair, D Donnai, T Andrews

Correspondence to Dr Andrews.

Received for publication 17 December 1990 Accepted for publication 31 December 1990.
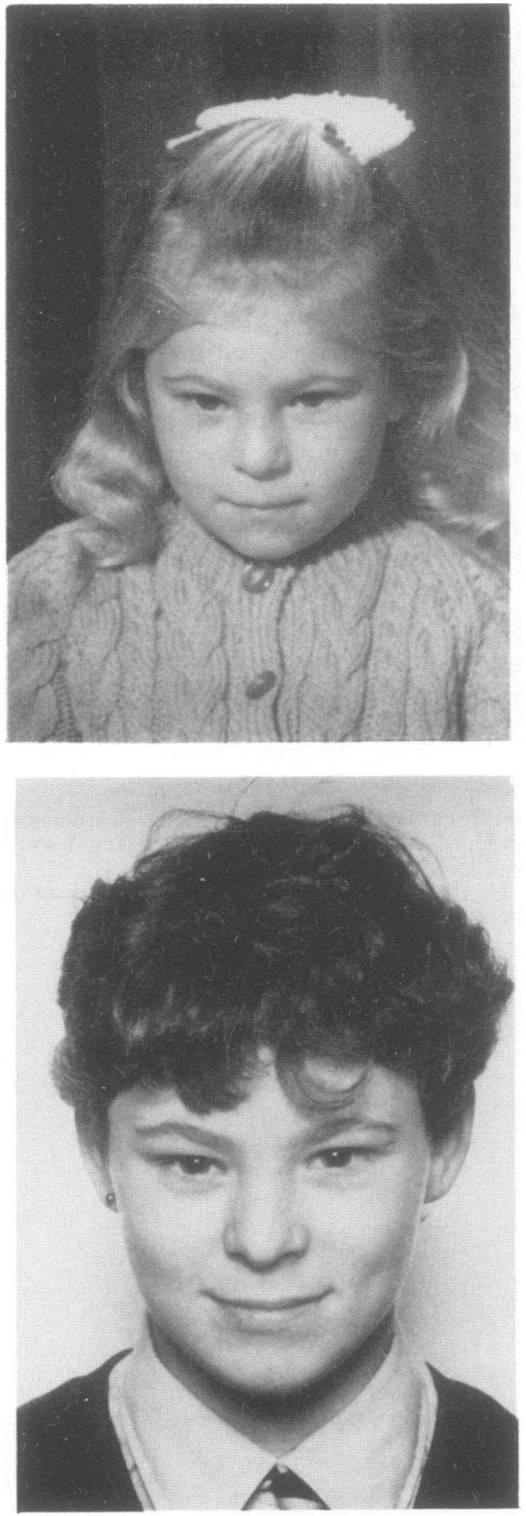

Figure 1 The facial appearance of the proband at 3 years (top) and 16 years (bottom). 


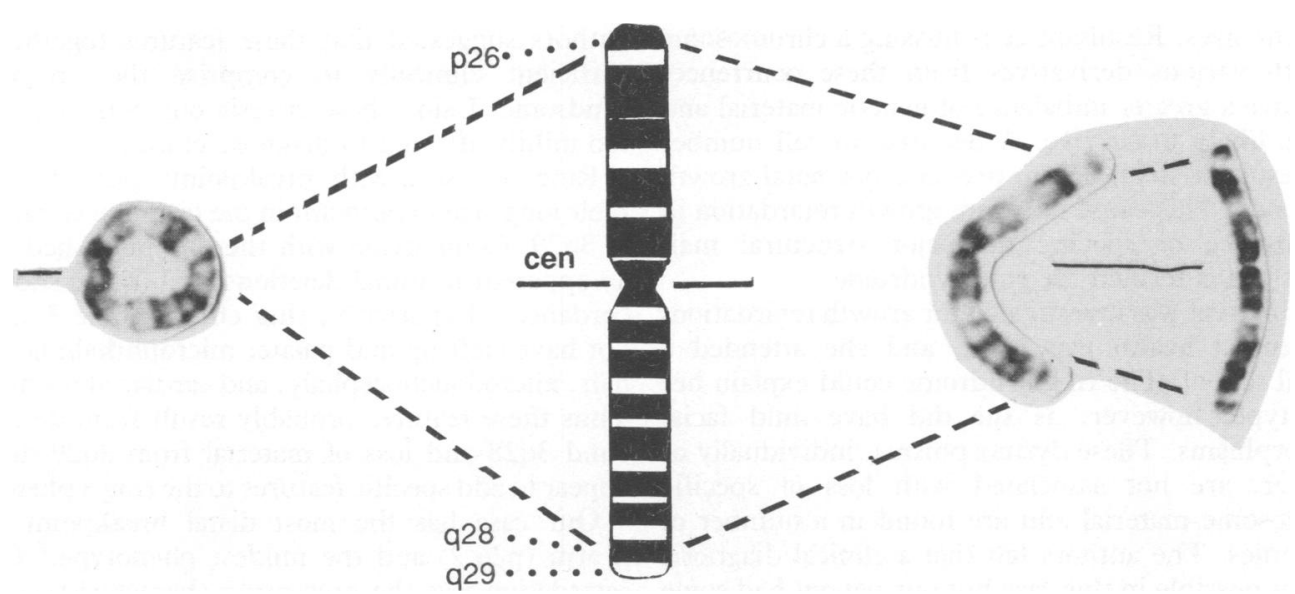

Figure 2 Partial karyotype showing GTL banded ring chromosome 3(left), compared with ideogram (centre) and two normal chromosomes 3 (right).

body proportions and Tanner stage 4 pubertal development. Her face was triangular with mild retrognathia and upward slanting palpebral fissures (fig 1). There was telecanthus, small alae nasi with a full nasal tip, and normally positioned, simple ears. Two small café au lait patches were noted on the medial aspect of her right thigh and she had syndactyly of toes 2 and 3 . There was mild limitation of extension of both elbows and short fifth fingers bilaterally.

\section{CYTOGENETIC ANALYSIS}

Chromosome preparations were obtained by routine lymphocyte culture with thymidine block and release. A total of $100 \mathrm{GTL}$ banded metaphases was analysed, the majority (84\%) of which were shown to have a $46, \mathrm{XX}, \mathrm{r}(3)(\mathrm{p} 26.2 \mathrm{q} 29)$ karyotype (figs 2 and 3). In addition, two cells with a 47, XX, r(3), $+\mathrm{r}(3)$ and four cells with various ring and rod breakage configurations

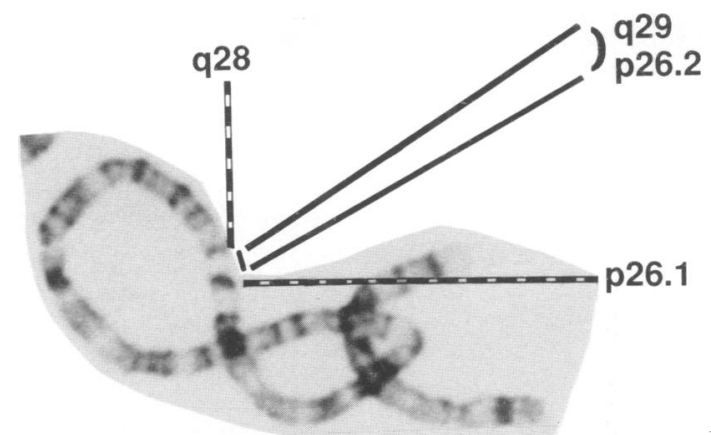

Figure 3 Partial karyotype showing high resolution GTL banding of the duplicated $r(3)$. Breakpoint in the short arm beyond the $G$ dark band at $p 26.1$ and in $G$ light band at $p 26.2$. Breakpoint in the long arm in $G$ light band at $q 29$. were observed. The remaining cells had a larger ring, thought to be a duplicated ring product. Further studies using CBG banding indicated that the $r(3)$ had a single centromere and that the larger ring product had two centromeres, consistent with a duplicated dicentric ring chromosome.

Comparison of the breakpoint in this case with those in the previously published reports indicated that the present case had the most distal breakpoint in the short arm (3p26.2) compared with $3 \mathrm{p} 26.1^{5}$ and 3 p26. ${ }^{34}$ The breakpoint in the long arm is apparently the same in all four cases (3q29).

Unfortunately, a cell line is not available from this patient.

\section{Discussion}

Our case is the sixth reported subject with ring chromosome 3 and the fourth in whom chromosome analysis defined the ring breakpoints. A ring autosome may result from end to end fusion of telomeres without obvious loss of genetic material, but in our case there was loss of material from both the long and short arms of chromosome 3.

The phenotype of reported subjects with ring chromosome 3 appeared non-specific with growth retardation, mild dysmorphism, and variable mental retardation. This raised the possibility that the phenotype was the result of the 'ring syndrome' that is common to all ring chromosome cases. Kosztolanyi ${ }^{6}$ analysed 207 case reports of subjects with a ring autosome and discussed the ring syndrome. He found the size of the ring correlated with its instability and postulated that sister chromatid exchanges within a ring produce secondary chromosome abnormalities, such as dicentric and interlocking rings. Also ring chromosomes may lag at anaphase and form configurations, such as anaphase bridges and rings of 
different sizes. Resultant cells missing a chromosome or with various derivatives from these ocurrences may have a greater imbalance of genetic material and be less likely to survive. A decrease in cell number may result predisposing to pre- and postnatal growth deficiency. This cause of severe growth retardation in the absence of specific or major structural malformations is termed the ring syndrome.

Our patient was investigated for growth retardation, her general health was good, and she attended a normal school. The ring syndrome could explain her phenotype, however, as she did have mild facial dysmorphisms. These dysmorphisms, individually or together, are not associated with loss of specific chromosome material and are found in a number of syndromes. The authors felt that a clinical diagnosis was not possible in this case but our patient had some features in common with other patients reported to have a deletion of distal 3p.

Wilson $e t a l^{3}$ reported a male child with karyotype $46, X Y, r 3(p 26-q 29)$, growth failure, developmental delay, and dysmorphic features. He was microcephalic with a flattened face, small palpebral fissures, ptosis, epicanthic folds, and a small mouth with downturned corners. The authors suggested a recognisable clinical syndrome could result from the deletion of 3p26-pter. However, some features of this child, such as redundant nuchal skin, hypoplastic nipples, hypospadias, and deep plantar creases, have not been reported in other cases of ring chromosome 3.

Narahara $e t a l$ reported a patient with karyotype $46, \mathrm{XX}, \operatorname{del}(3)(\mathrm{p} 25.3)$ and reviewed the previously reported cases with a deletion of the distal portion of $3 p$. They concluded that loss of the band 3p25.3 was critical in producing a clinically recognisable phenotype. In addition to the growth and mental retardation and mild dysmorphism, loss of band 3p25.3 was likely to have produced microcephaly, blepharoptosis, ear malformations, polydactyly, synophrys, and bushy eyebrows, and less often genitourinary malformations, cardiovascular anomalies, and rockerbottom feet. The authors suggested that these features together were sufficient clinically to comprise the $3 p$ deletion syndrome. Using these criteria our patient would be too mildly affected to diagnose clinically.

Ring 3 cases, with breakpoints published, have their long arm breakpoint in the terminal G light band at 3q29. Comparison with the one published case of an apparent terminal deletion $\operatorname{del}(3)$ (q28:) shows discordance. ${ }^{7}$ Reportedly, ring chromosome 3 cases do not have cleft lip and palate, microphthalmia, scanty hair, microdolichocephaly, and cardiac abnormalities. Thus these features probably result from deletion of band 3q28 and loss of material from 3q29 does not appear to add specific features to the ring 3 phenotype.

Our case has the most distal breakpoint in the $p$ arm (p26.2) and the mildest phenotype. ${ }^{8}$ Growth retardation was the presenting characteristic and the only cause of parental concern. Her mild dysmorphisms were not recognisable as a distinct syndrome. We conclude that her overall phenotype could be explained by the ring syndrome although her facial dysmorphisms are found in ring chromosome 3 cases with a more proximal short arm breakpoint.

1 Picciano D, Berlin C, Davenport S, et al. Human ring chromosomes: a report of five cases. Ann Genet (Paris) 1972;15:241-7.

2 Witkowski R, Ultrich E, Piede U. Ring chromosome 3 in a retarded boy. Hum Genet 1978;42:354-8.

3 Wilson G, Pooley J, Parker J. The phenotype of ring chromosome 3. F Med Genet 1982;19:471-3.

4 Katitani M, Takahashi $\mathrm{H}$, Yasuda J, et al. A case of ring chromosome 3, 46,XX,-3,+r(3)(p26q29). Jpn f Hum Genet 1984;29:157-62.

5 Narahara K, Kikkawa K, Murakami M, et al. Loss of the 3p25.3 band is critical in the manifestation of del(3p) syndrome: karyotype-phenotype correlation in cases with deficiency of the distal portion of the short arm of chromosome 3. Am 7 Med Genet 1990;35:269-73.

6 Kosztolanyi G. Does "ring syndrome" exist? An analysis of 207 case reports on patients with a ring autosome. Hum Genet 1987;75:174-9.

7 Alvarez Arratia $M$, Rivera $H$, Moller $M$, et al. De novo $\operatorname{del}(3)(\mathrm{q} 2800)$. Ann Genet (Paris) 1984;27:109-11.

8 Tolmie J, Batstone P, Ruthven I, Gilmore D. Partial deletion of the short arm of chromosome 3. Clin Genet 1986;29:538-42. 